\title{
CORE-COLLAPSE SUPERNOVAE FROM THE PALOMAR TRANSIENT FACTORY: INDICATIONS FOR A DIFFERENT POPULATION IN DWARF GALAXIES
}

Iair Arcavi ${ }^{1}$, Avishay Gal-Yam ${ }^{1}$, Mansi M. Kasliwal ${ }^{2}$, Robert M. Quimby ${ }^{2}$, Eran O. Ofek ${ }^{2}$, Shrinivas R. Kulkarni $^{2}$, Peter E. Nugent ${ }^{3}$, S. Bradley Cenko ${ }^{4}$, Joshua S. Bloom ${ }^{4}$, Mark Sullivan $^{5}$, D. Andrew Howell ${ }^{6,7}$, Dovi Poznanski ${ }^{3,4,11}$,

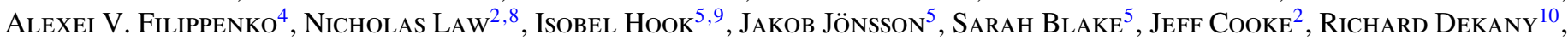
Gustavo Rahmer ${ }^{10}$, David Hale ${ }^{10}$, Roger Smith ${ }^{10}$, JefF Zolkower ${ }^{10}$, Viswa Velur ${ }^{10}$, Richard Walters ${ }^{10}$, JoHn Henning ${ }^{10}$, KAHnh Bui ${ }^{10}$, DAN MCKennA ${ }^{10}$, AND JANET JACOBSEN ${ }^{3}$

${ }^{1}$ Benoziyo Center for Astrophysics, Faculty of Physics, The Weizmann Institute of Science, Rehovot 76100, Israel; iair.arcavi@weizmann.ac.il

${ }^{2}$ Cahill Center for Astrophysics, California Institute of Technology, Pasadena, CA 91125, USA

${ }^{3}$ Computational Cosmology Center, Lawrence Berkeley National Laboratory, 1 Cyclotron Road, Berkeley, CA 94720, USA

${ }^{4}$ Department of Astronomy, University of California, Berkeley, CA 94720-3411, USA

${ }^{5}$ Department of Physics (Astrophysics), University of Oxford, Keble Road, Oxford, OX1 3RH, UK

${ }^{6}$ Las Cumbres Observatory Global Telescope Network, 6740 Cortona Dr., Suite 102, Goleta, CA 93117, USA

${ }^{7}$ Department of Physics, University of California, Santa Barbara, Broida Hall, Mail Code 9530, Santa Barbara, CA 93106-9530, USA

${ }^{8}$ Dunlap Institute for Astronomy and Astrophysics, University of Toronto, 50 St. George Street, Toronto M5S 3H4, Ontario, Canada

${ }^{9}$ INAF-Osservatorio Astronomico di Roma, via Frascati 33, 00040 Monteporzio (RM), Italy

${ }^{10}$ Caltech Optical Observatories, California Institute of Technology, Pasadena, CA 91125, USA Received 2010 April 16; accepted 2010 July 26; published 2010 September 1

\begin{abstract}
We use the first compilation of 72 core-collapse supernovae (SNe) from the Palomar Transient Factory (PTF) to study their observed subtype distribution in dwarf galaxies compared to giant galaxies. Our sample is the largest single-survey, untargeted, spectroscopically classified, homogeneous collection of core-collapse events ever assembled, spanning a wide host-galaxy luminosity range (down to $M_{r} \approx-14 \mathrm{mag}$ ) and including a substantial fraction ( $>20 \%)$ of dwarf $\left(M_{r} \geqslant-18 \mathrm{mag}\right.$ ) hosts. We find more core-collapse SNe in dwarf galaxies than expected and several interesting trends emerge. We use detailed subclassifications of stripped-envelope core-collapse $\mathrm{SNe}$ and find that all Type I core-collapse events occurring in dwarf galaxies are either $\mathrm{SNe} \mathrm{Ib}$ or broad-lined $\mathrm{SNe} \mathrm{Ic}$ (SNe Ic-BL), while "normal" SNe Ic dominate in giant galaxies. We also see a significant excess of SNe IIb in dwarf hosts. We hypothesize that in lower metallicity hosts, metallicity-driven mass loss is reduced, allowing massive stars that would have appeared as "normal" SNe Ic in metal-rich galaxies to retain some He and H, exploding as $\mathrm{Ib} / \mathrm{IIb}$ events. At the same time, another mechanism allows some stars to undergo extensive stripping and explode as SNe Ic-BL (and presumably also as long-duration gamma-ray bursts). Our results are still limited by small-number statistics, and our measurements of the observed $N(\mathrm{Ib} / \mathrm{c}) / N$ (II) number ratio in dwarf and giant hosts $\left(0.25_{-0.15}^{+0.3}\right.$ and $0.23_{-0.08}^{+0.11}$, respectively; $1 \sigma$ uncertainties) are consistent with previous studies and theoretical predictions. As additional PTF data accumulate, more robust statistical analyses will be possible, allowing the evolution of massive stars to be probed via the dwarf-galaxy SN population.
\end{abstract}

Key words: supernovae: general

Online-only material: color figures

\section{INTRODUCTION}

Understanding the formation, evolution, and ultimate fate of massive stars is a key open question in astrophysics. A particularly interesting problem concerns the formation of highly stripped objects known as Wolf-Rayet (W-R) stars. It is still unclear whether W-R stars are formed through winddriven mass loss from single very massive stars or if binary interactions play an important role in which case the minimal initial mass of W-R stars may be much lower. The final stages in the evolution of a massive star influence the type of explosion it will undergo at the end of its life; studying the resulting core-collapse supernovae ( $\mathrm{SNe}$ ) is thus an attractive way to learn about these massive progenitors. The hope is to reveal how the physical properties of the star (such as its mass and metallicity) determine its pre-explosion mass loss, as reflected in the properties of the ensuing SN. An additional benefit is that luminous SN explosions are visible to great distances and hence

\footnotetext{
${ }^{11}$ Einstein fellow.
}

provide access to a wide range of stellar populations, while normal individual stars can be effectively studied only within the Local Group.

Core-collapse SN explosions of massive stars are observationally classified according to their spectroscopic properties (see Filippenko 1997 for a review). H-rich Type IIP ("plateau") SNe have been associated with red supergiant progenitors (Smartt 2009 and references therein). The progenitors of Type IIb SNe appear to have lost most (but not all) of their hydrogen envelope before they exploded. Further mass loss leads to Type $\mathrm{Ib}$ explosions showing strong $\mathrm{He}$ lines but lacking $\mathrm{H}$, while $\mathrm{SNe}$ Ic, lacking both $\mathrm{H}$ and $\mathrm{He}$, probably arise from the most highly stripped progenitors. A particular subclass of SNe Ic, displaying very broad lines (SNe Ic-BL) indicating high expansion velocities, is notable for the fact that its members are the only events to have been observationally linked with gamma-ray bursts (GRBs) and X-ray flashes (XRFs; see Woosley \& Bloom 2006 for a review). While it stands to reason that $\mathrm{H}$-poor $\mathrm{SNe}$ arise from H-poor W-R stars, attempts to directly detect such stars in pre-explosion images of the locations of $\mathrm{SNe} \mathrm{Ib}$ and 
Ic have so far failed (Gal-Yam et al. 2005; Maund et al. 2005; Crockett et al. 2008b; Smartt 2009). The exact identity of the progenitor stars of $\mathrm{SNe} \mathrm{IIb} / \mathrm{Ib} / \mathrm{Ic}$, as well as those of GRBs and XRFs, remains an unsolved puzzle.

Given the limited efficacy of direct pre-explosion imaging studies, one can attempt to study massive-star populations using statistical analyses of the resulting SNe. Two main methods have been employed for this purpose thus far as follows.

The number ratio of $\mathrm{SNe} \mathrm{Ib} / \mathrm{c}$ to $\mathrm{SNe}$ II as a function of hostgalaxy metallicity has been measured using host luminosity as a metallicity proxy for 280 and 497 core-collapse $\mathrm{SNe}$ (Prantzos \& Boissier 2003 and Boissier \& Prantzos 2009, respectively), as well as via direct metallicity measurements for 159 corecollapse SNe (Prieto et al. 2008). Both methods show that the ratio of $\mathrm{SNe} \mathrm{Ib} / \mathrm{c}$ to $\mathrm{SNe}$ II increases with increasing host metallicity. This suggests that wind-driven mass loss plays an important role in the formation of stripped W-R stars exploding as $\mathrm{SNe} \mathrm{Ib} / \mathrm{c}$ and that at lower metallicities massive stars that would have ended as $\mathrm{SNe} \mathrm{Ib} / \mathrm{c}$ explode instead as Type II events ("failed Ib/c"). Heger et al. (2003) predicted this behavior, but their decline rate for the $\mathrm{SN} \mathrm{Ib} / \mathrm{c}$ ratio with decreasing metallicity, as well as that of Fryer et al. (2007), does not agree with the Boissier \& Prantzos (2009) data.

The location of a SN in its host galaxy can also be indicative of the mass and metallicity of its progenitor. Anderson \& James (2009) examined 173 core-collapse SN locations and confirmed previous results by van den Bergh (1997), Tsvetkov et al. (2004), and Hakobyan (2008) which indicated that SNe Ib/c are preferentially found in more central regions of their host galaxies compared to SNe II (see also Boissier \& Prantzos 2009; Hakobyan et al. 2009), with SNe Ic the most centrally concentrated. This implies a progenitor-metallicity sequence (II-Ib-Ic) with SNe Ic having the highest metallicity progenitors. Like the population-ratio studies, these results also suggest that $\mathrm{SN} \mathrm{Ib/c}$ progenitor analogs (in terms of mass) would explode as SNe II in low-metallicity regions. Other location studies, however, indicate otherwise. Fruchter et al. (2006) found that long-duration GRBs occur preferentially in faint (metal-poor) galaxies (see also Stanek et al. 2006) and in brighter regions than the general core-collapse population (based on 16 core-collapse SN locations). Kelly et al. (2008) showed that SNe Ic (26 in their sample) occur in areas within their hosts similar to those of long-duration GRBs. Taken together, these results, as well as those of Leloudas et al. (2010), would suggest that some SN Ic progenitor analogs in low-metallicity environments tend to explode as SNe Ic-BL (accompanied by long GRBs) rather than as $\mathrm{SNe}$ II.

While the results from the studies described above are intriguing and hint at the power of the statistical analysis of large SN samples, a clear picture has yet to emerge. Perhaps this is due to the small number of events in dwarf, low-metallicity galaxies (leading Prantzos \& Boissier 2003 and Boissier \& Prantzos 2009, for example, to extend the "dwarf" galaxy bin to luminosities as bright as $-19 \mathrm{mag}$ ). It should also be noted that many studies to date were based on heterogeneous compilations of SN data, inheriting their strong and unknown selection effects, and many included $\mathrm{SNe}$ discovered decades ago, the exact classification of which was unclear (Anderson \& James 2009). Here, we present a population-ratio analysis for the first sample of 72 core-collapse SNe from the Palomar Transient Factory (PTF; Rau et al. 2009; Law et al. 2009), 15 of which are in dwarf galaxies having $r$-band magnitudes $M_{r} \geqslant-18$. The observational data are described in Section 2 and the results in
Section 3. We discuss our findings in Section 4 and summarize in Section 5.

\section{OBSERVATIONS}

\subsection{The Palomar Transient Factory}

PTF is a wide-field variability survey aimed at a systematic study of the transient sky. The survey camera, mounted on the 48 in Oschin Schmidt Telescope at Palomar Observatory (P48), delivers a $7.8 \mathrm{deg}^{2}$ field of view per image. The survey is now fully operational, having discovered thousands of transients and hundreds of SNe. See Rau et al. (2009) for a review of PTF science and observing strategies and Law et al. (2009) for performance and technical information concerning PTF.

One of the key objectives of PTF involves the construction of a large sample of core-collapse events, for which multicolor optical light curves and spectra are collected through dedicated follow-up resources. Such data will allow for a systematic study of the core-collapse SN population, the first results of which are presented here.

\subsection{SN Sample}

Our SN sample is comprised of 72 nearby (redshift $z=$ $0.008-0.184$, with mean 0.056 and median 0.046; Figure 1) core-collapse SNe discovered by PTF (Table 1; discussed in detail by A. Gal-Yam et al. 2010, in preparation) from 2009 March through 2010 March. PTF provides an untargeted (i.e., random across the sky, unlike previous SN surveys which focused on known, generally large, galaxies), homogeneous sample of core-collapse events. The high-cadence PTF monitoring and its sensitivity largely reduce effects of light-curve shape and type-specific peak absolute magnitudes in biasing against a certain variety of SN. While the PTF detection efficiencies have not yet been fully quantified, any type-specific biases should not depend on host-galaxy luminosity and are therefore unlikely to impact our analysis. The sample analyzed here includes only events spectroscopically confirmed as core-collapse SNe. ${ }^{12}$

While smaller than the data sets used in previous studies, the nature of the PTF survey provides a minimally biased homogeneous sample. To our knowledge, it is the largest collection of core-collapse events ever assembled from a singleuntargeted survey, rich $(>20 \%)$ in $\mathrm{SNe}$ from dwarf $\left(M_{r} \geqslant\right.$ $-18 \mathrm{mag}$ ) hosts. Furthermore, a detailed subtype classification has been carried out for all of the $\mathrm{SNe}$ in our sample.

\subsection{Classification}

All SN events were spectroscopically confirmed using either the Low Resolution Imaging Spectrometer (LRIS; Oke et al. 1995) mounted on the Keck I 10 m telescope, the DEep Imaging Multi-Object Spectrograph (DEIMOS; Faber et al. 2003) mounted on the Keck II $10 \mathrm{~m}$ telescope, the double spectrograph (DBSP; Oke \& Gunn 1982) mounted on the Palomar $5 \mathrm{~m}$ telescope, the Intermediate dispersion Spectrograph and Imaging System (ISIS) mounted on the William Herschel $4.2 \mathrm{~m}$ telescope, the Kast spectrograph (Miller \& Stone 1993) mounted on the Shane $3 \mathrm{~m}$ telescope, the Gemini Multi-Object Spectrograph (GMOS; Hook et al. 2004) mounted on the Gemini North

\footnotetext{
12 Here, we exclude new subclasses for which only a few examples have been identified, such as pair-production events (Gal-Yam et al. 2009), very luminous SNe (Quimby et al. 2009), Type Ibn SNe (Foley et al. 2007; Pastorello et al. 2008a), SN 2002cx-like events (Li et al. 2003), and SN 2002ic-like events (Hamuy et al. 2003).
} 
Table 1

PTF Core-collapse SNe, 2009 March through 2010 March $^{\mathrm{a}}$

\begin{tabular}{|c|c|c|c|c|c|c|}
\hline Name & Type & $\alpha(\mathrm{J} 2000)$ & $\delta(\mathbf{J} 2000)$ & $\begin{array}{l}\text { Host } m_{r} \\
\text { (mag) }\end{array}$ & $z$ & $\begin{array}{l}\text { Host } M_{r} \\
\text { (mag) }\end{array}$ \\
\hline PTF09aux & SN Ic/Ia & 16:09:15.84 & $+29: 17: 36.7$ & 16.14 & 0.047 & -20.56 \\
\hline PTF09awk & SN Ib & $13: 37: 56.36$ & $+22: 55: 04.8$ & 17.70 & 0.062 & -19.58 \\
\hline PTF09axc & SN II & $14: 53: 13.06$ & $+22: 14: 32.2$ & 18.12 & 0.115 & -20.61 \\
\hline PTF09axi & SN II & $14: 12: 40.82$ & $+31: 04: 03.3$ & 18.42 & 0.064 & -18.91 \\
\hline PTF09bce & SN II & $16: 35: 17.66$ & $+55: 37: 59.1$ & $\mathrm{n} / \mathrm{a}$ & 0.023 & $\mathrm{n} / \mathrm{a}$ \\
\hline PTF09bcl & SN II & 18:06:26.78 & $+17: 51: 43.0$ & 16.23 & 0.062 & -21.23 \\
\hline PTF09be & SN II & $14: 10: 18.53$ & $+16: 53: 39.0$ & $>21.00$ & 0.09 & $>-17.13$ \\
\hline PTF09bgf & SN II & $14: 41: 38.28$ & $+19: 21: 43.8$ & 17.99 & 0.031 & -17.75 \\
\hline PTF09bw & SN II & $15: 05: 02.04$ & $+48: 40: 01.9$ & 20.28 & 0.15 & -19.04 \\
\hline PTF09cjq & SN II & $21: 16: 28.48$ & $-00: 49: 39.7$ & 13.23 & 0.019 & -21.53 \\
\hline PTF09ct & SN II & $11: 42: 13.80$ & $+10: 38: 53.9$ & 19.38 & 0.15 & -19.99 \\
\hline PTF09cu & SN II & $13: 15: 23.14$ & $+46: 25: 08.6$ & 15.47 & 0.057 & -21.60 \\
\hline PTF09cvi & SN II & 21:47:09.80 & $+08: 18: 35.6$ & 19.40 & 0.03 & -16.41 \\
\hline PTF09dah & SN IIb & $22: 45: 17.05$ & $+21: 49: 15.2$ & 16.92 & 0.0238 & -18.32 \\
\hline PTF09dfk & $\mathrm{SN} \mathrm{Ib}$ & 23:09:13.42 & $+07: 48: 15.4$ & 16.90 & 0.016 & -17.45 \\
\hline PTF09dj1 & SN II & $16: 33: 55.94$ & $+30: 14: 16.3$ & 19.74 & 0.184 & -20.07 \\
\hline PTF09dra & SN II & $15: 48: 11.47$ & $+41: 13: 28.2$ & 16.54 & 0.077 & -21.22 \\
\hline PTF09due & SN II & $16: 26: 52.36$ & $+51: 33: 23.9$ & 13.79 & 0.029 & -21.78 \\
\hline PTF09dxv & SN IIb & $23: 08: 34.73$ & $+18: 56: 13.7$ & 16.14 & 0.033 & -20.13 \\
\hline PTF09dzt & SN Ic & $16: 03: 04.20$ & $+21: 01: 47.2$ & 17.17 & 0.0874 & -21.09 \\
\hline PTF09ebq & SN II & 00:14:01.69 & $+29: 25: 58.5$ & 15.15 & 0.0235 & -20.01 \\
\hline PTF09ecm & SN II & 01:06:43.16 & $-06: 22: 40.9$ & 16.52 & 0.0285 & -19.35 \\
\hline PTF09ejz & SN Ic/Ia & 00:55:07.29 & $-06: 57: 05.4$ & 16.93 & 0.11 & -21.87 \\
\hline PTF09fae & SN IIb & $17: 26: 20.33$ & $+72: 56: 30.6$ & 19.78 & 0.067 & -17.72 \\
\hline PTF09fbf & SN II & $21: 20: 38.44$ & $+01: 02: 52.9$ & 14.70 & 0.021 & -20.31 \\
\hline PTF09fma & SN II & $03: 10: 23.33$ & $-09: 59: 58.0$ & 16.17 & 0.031 & -19.74 \\
\hline PTF09fmk & SN II & $23: 57: 46.19$ & $+11: 58: 45.3$ & 16.48 & 0.0631 & -21.02 \\
\hline PTF09foq & SN II & $05: 18: 54.83$ & $+19: 10: 11.4$ & 15.42 & 0.02 & -20.62 \\
\hline PTF09foy & SN II & $23: 17: 10.58$ & $+17: 15: 03.2$ & 16.38 & 0.06 & -20.88 \\
\hline PTF09fqa & SN II & $22: 25: 32.33$ & $+18: 59: 41.4$ & 16.17 & 0.03 & -19.55 \\
\hline PTF09fsr ${ }^{b}$ & $\mathrm{SN} \mathrm{Ib}$ & 23:04:52.98 & $+12: 19: 59.0$ & 10.74 & 0.007942 & -22.23 \\
\hline PTF09g & SN II & $15: 16: 31.48$ & $+54: 27: 34.7$ & 15.99 & 0.04 & -20.29 \\
\hline PTF09gof & SN II & $01: 22: 25.60$ & $+03: 38: 08.4$ & 17.65 & 0.103 & -20.82 \\
\hline PTF09gpn & SN II & $03: 43: 43.26$ & $-17: 08: 43.1$ & 19.13 & 0.015 & -15.36 \\
\hline PTF09gtt & SN II & $02: 20: 37.70$ & $+02: 24: 13.2$ & 18.68 & 0.041 & -17.72 \\
\hline PTF09gyp & SN IIb & 01:58:56.76 & $-07: 16: 56.9$ & 21.75 & 0.046 & -14.87 \\
\hline PTF09hdo & SN II & $00: 15: 23.20$ & $+30: 43: 19.3$ & 14.79 & 0.047 & -22.00 \\
\hline PTF09hzg & SN II & $11: 50: 57.74$ & $+21: 11: 49.4$ & 15.43 & 0.028 & -20.12 \\
\hline PTF09iex & SN II & $12: 02: 46.86$ & $+02: 24: 06.8$ & 18.40 & 0.02 & -16.37 \\
\hline PTF09ige & SN II & $08: 55: 34.24$ & $+32: 39: 57.0$ & 17.10 & 0.064 & -20.27 \\
\hline PTF09igz & SN II & 08:53:56.70 & $+33: 40: 11.5$ & 18.77 & 0.086 & -19.27 \\
\hline PTF09iqd & SN Ic & $02: 35: 23.23$ & $+40: 17: 08.7$ & 14.93 & 0.034 & -21.11 \\
\hline PTF09ism & SN II & $11: 44: 35.87$ & $+10: 12: 43.7$ & 17.27 & 0.029 & -18.46 \\
\hline PTF09ps & SN Ic & $16: 14: 08.62$ & $+55: 41: 41.4$ & 19.52 & 0.1065 & -18.96 \\
\hline PTF09q & SN Ic & $12: 24: 50.11$ & $+08: 25: 58.8$ & 16.61 & 0.09 & -21.53 \\
\hline PTF09r & SN II & $14: 18: 58.63$ & $+35: 23: 16.0$ & 17.87 & 0.027 & -17.53 \\
\hline PTF09sh & SN II & $16: 13: 58.08$ & $+39: 31: 58.1$ & 15.35 & 0.0377 & -20.78 \\
\hline PTF09sk & SN Ic-BL & $13: 30: 51.15$ & $+30: 20: 04.9$ & 17.43 & 0.0355 & -18.57 \\
\hline PTF09t & SN II & $14: 15: 43.29$ & $+16: 11: 59.1$ & 16.05 & 0.039 & -20.17 \\
\hline PTF09tm & SN II & $13: 46: 55.94$ & $+61: 33: 15.6$ & 15.33 & 0.035 & -20.66 \\
\hline PTF09uj & SN II & $14: 20: 11.15$ & $+53: 33: 41.0$ & 17.27 & 0.0651 & -20.09 \\
\hline PTF10bau & SN II & $09: 16: 21.29$ & $+17: 43: 40.2$ & 14.03 & 0.026 & -21.33 \\
\hline PTF10bfz & SN Ic-BL & $12: 54: 41.27$ & $+15: 24: 17.0$ & 21.90 & 0.15 & -17.42 \\
\hline PTF10bgl & SN II & 10:19:04.70 & $+46: 27: 23.3$ & 13.63 & 0.03 & -22.00 \\
\hline PTF10bhu & SN Ic & $12: 55: 28.44$ & $+53: 34: 28.7$ & 16.63 & 0.036 & -19.40 \\
\hline PTF10bip & SN Ic & $12: 34: 10.52$ & $+08: 21: 48.5$ & 18.64 & 0.051 & -18.19 \\
\hline PTF10bzf ${ }^{c}$ & SN Ic-BL & 11:44:02.99 & $+55: 41: 27.6$ & 19.05 & 0.0498 & -17.71 \\
\hline PTF10cd & SN II & 03:00:32.93 & $+36: 15: 25.4$ & 19.08 & 0.0455 & -18.00 \\
\hline PTF10con & SN II & $16: 11: 39.09$ & $+00: 52: 33.3$ & 16.78 & 0.033 & -19.36 \\
\hline PTF10cqh & SN II & $16: 10: 37.60$ & $-01: 43: 00.7$ & 15.12 & 0.041 & -21.57 \\
\hline PTF10cwx & SN II & $12: 33: 16.53$ & $-00: 03: 10.6$ & 18.52 & 0.073 & -19.14 \\
\hline PTF10cxq & SN II & $13: 48: 19.32$ & $+13: 28: 58.8$ & 17.55 & 0.047 & -19.11 \\
\hline PTF10cxx & SN II & $14: 47: 27.78$ & $+01: 55: 03.8$ & 15.79 & 0.034 & -20.20 \\
\hline PTF10czn & SN II & $14: 51: 16.23$ & $+15: 26: 43.6$ & 14.97 & 0.045 & -21.63 \\
\hline PTF10dk & SN II & $05: 08: 21.54$ & $+00: 12: 42.9$ & 23.55 & 0.074 & -14.28 \\
\hline
\end{tabular}


Table 1

(Continued)

\begin{tabular}{lccccc}
\hline \hline \multicolumn{1}{c}{ Name } & Type & $\alpha(\mathrm{J} 2000)$ & $\delta(\mathrm{J} 2000)$ & $\begin{array}{c}\text { Host } m_{r} \\
(\mathrm{mag})\end{array}$ & $\begin{array}{c}z \\
\text { Host } M_{r} \\
(\mathrm{mag})\end{array}$ \\
\hline PTF10dvb $^{\text {d }}$ & SN II & $17: 16: 12.25$ & $31: 47: 36.0$ & 14.43 & 0.022942 \\
PTF10hv & SN II & $14: 03: 56.18$ & $+54: 27: 31.1$ & 16.93 & 0.0518 \\
PTF10in & SN IIb & $07: 50: 01.24$ & $+33: 06: 23.8$ & 20.46 & 0.07 \\
PTF10s & SN II & $10: 37: 16.30$ & $+38: 06: 23.2$ & 17.42 & 0.051 \\
PTF10ts & SN II & $12: 33: 56.40$ & $+13: 55: 08.3$ & 16.84 & 0.046 \\
PTF10u & SN II & $10: 09: 58.42$ & $+46: 00: 35.2$ & 19.83 & -19.91 \\
PTF10wg & SN II/Ib/c & $03: 31: 45.78$ & $-25: 24: 03.3$ & $>21.00$ & -19 \\
\hline
\end{tabular}

Notes.

a Two SNe were omitted: PTF10wg due to a classification problem and PTF09bce because the presence of two overlapping galaxies along the line of sight prevented a unique host determination. Redshift values have varying accuracy due to varying resolution and quality of the spectra.

b SN2009jf.

c SN2010ah.

d SN2010aw.

e SN2009nn.

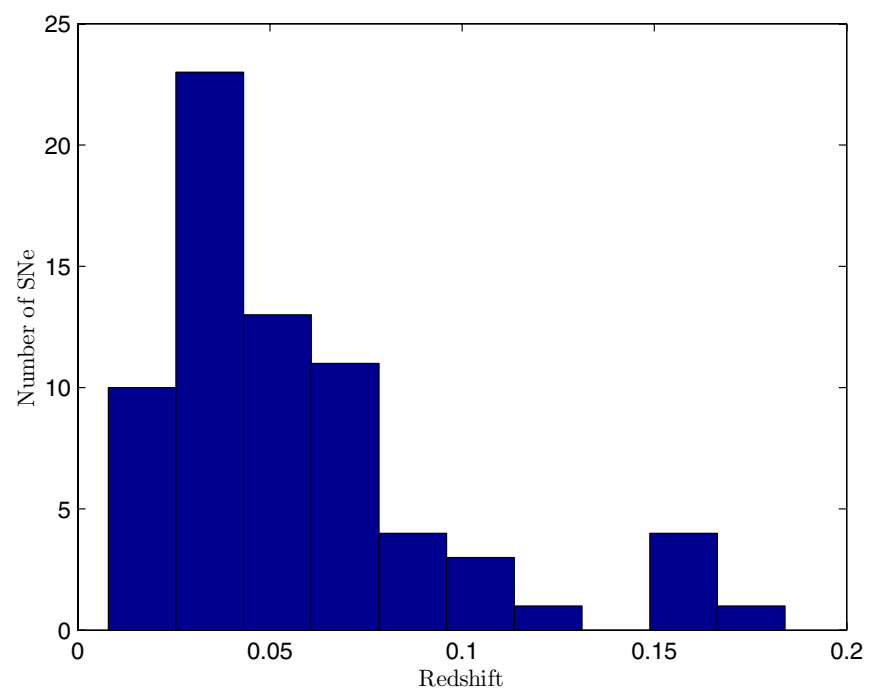

Figure 1. Redshift distribution of our sample of $72 \mathrm{SNe}$. (A color version of this figure is available in the online journal.)

$8 \mathrm{~m}$ telescope, or the X-Shooter spectrograph (D'Odorico et al. 2006) mounted on the Kueyen $8.2 \mathrm{~m}$ unit of the Very Large Telescope (VLT).

Events deemed most likely to be core-collapse $\mathrm{SNe}$ were classified (A. Gal-Yam et al. 2010, in preparation; Figure 2) as Type II (prominent $\mathrm{H}$ lines), IIb ( $\mathrm{H}$ lines as well as prominent $\mathrm{He}$ lines), $\mathrm{Ib}$ (no $\mathrm{H}$ and strong He lines), and $\mathrm{Ic}$ (no $\mathrm{H}$ or $\mathrm{He}$ lines). Both visual and automatic classifications, using Superfit (Howell et al. 2005) and SNID (Blondin \& Tonry 2007), were carried out. If a better match was found to SN Ic-BL templates (mainly SN 1998bw and SN 2002ap), the event was classified as a SN Ic-BL. Note that for the purpose of this paper, we do not distinguish between the different SN II subtypes (IIn, displaying narrow- and intermediate-width components; IIP/L, based on light-curve shape) except for Type IIb. This is in accord with direct detection of H-rich progenitors for SNe IIP and IIn (GalYam et al. 2007; Gal-Yam \& Leonard 2009; Smartt 2009), while analysis of the recent SN IIb 2008ax (Crockett et al. 2008a; Pastorello et al. 2008b; Chornock et al. 2010) may indicate a stripped progenitor (Crockett et al. 2008a). We consider Type II (H-rich), IIb (H-poor), Ib (H-less), and Ic (H- and He-less)

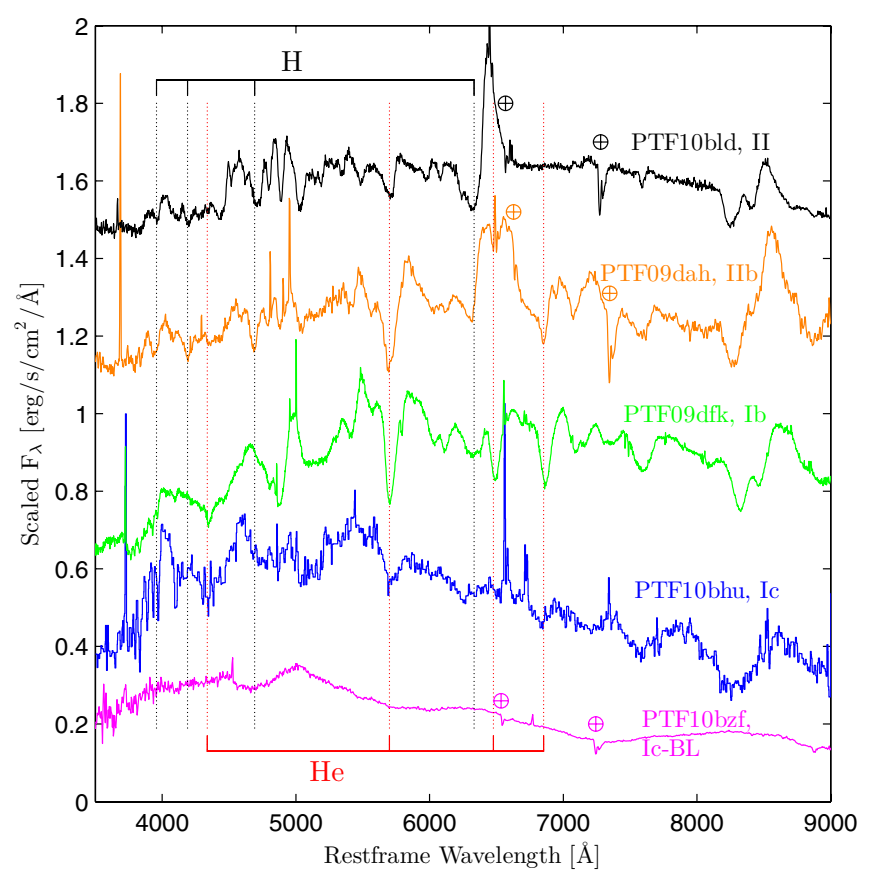

Figure 2. Spectroscopic classification. From top to bottom: a normal SN II (spectrum taken 12 days after peak) exhibits a strong Balmer hydrogen series and lacks prominent $\mathrm{He}$ I lines, especially redward of $\mathrm{H} \alpha$. In contrast, Type IIb events (spectrum taken 30 days after peak) show both hydrogen Balmer lines and the He I absorption series; note the typical absorption "notch" from $\mathrm{He}$ I $\lambda 6678$ superposed on the broad $\mathrm{H} \alpha$ emission profile. SNe Ib (spectrum taken approximately 35 days after peak) show strong He I lines but lack obvious $\mathrm{H}$ signatures, while in $\mathrm{SNe}$ Ic (spectrum taken about 18 days after peak) the He I lines are weak or absent. The bottom spectrum (spectrum taken some 3 days before peak) shows a broad-lined Type Ic event (SN Ic-BL); note the smooth shape due to extreme broadening of the lines (e.g., compare the Ca II near-infrared triplet profile with that in previous spectra). Data shown were obtained using Keck+LRIS (PTF10bld and PTF09dfk), P200+DBSP (PTF09dah), WHT+ISIS (PTF10bhu), and Gemini North+GMOS (PTF10bzf). The main telluric absorption features are marked.

(A color version of this figure is available in the online journal.)

as resulting from a progression of progenitors with increasingly intensive mass loss.

The spectra of two events (PTF09aux and PTF09ejz) were consistent with both SN Ia and SN Ic classifications. For statistical purposes, each was counted as half a Ic event. For 

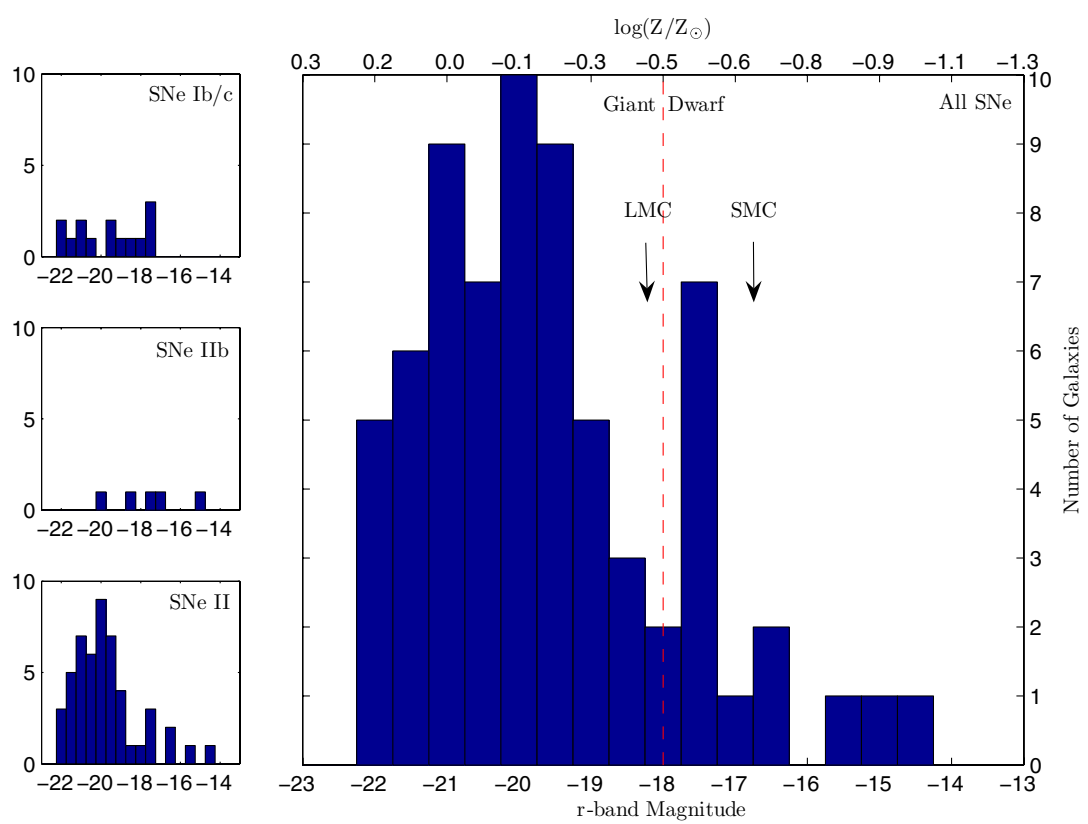

Figure 3. $r$-band absolute magnitudes of the host galaxies of our $72 \mathrm{SNe}$, except two for which only upper limits are available, and one for which a host could not be determined. The dwarf/giant cut we adopt is represented by the dashed line, and metallicity estimates are noted in the upper axis. The $r$-band magnitudes of the Large and Small Magellanic Clouds are also noted, converted from NED using the Smith et al. (2002) equations with distance moduli adopted from Keller \& Wood (2006). Host-magnitude histograms for different SN types are shown on left. SNe Ib/c are seen to be concentrated in luminous hosts. SNe IIb are located in the intermediate hosts and the SN II population extends toward the dimmest galaxies, as expected from metallicity considerations. An excess of SNe II in giant hosts compared to dwarf hosts is also evident.

(A color version of this figure is available in the online journal.)

one event (PTF10wg), the type of core-collapse SN could not be reliably determined, and so it was omitted from the study.

\subsection{Galaxy Luminosities}

Galaxy luminosities were taken from the Sloan Digital Sky Survey (SDSS) Data Release 7 (Abazajian et al. 2009), when available. For those hosts without SDSS coverage, pre-explosion images taken by the PTF camera on the P48 were constructed, and $r$-band magnitudes were measured using aperture photometry, calibrated against USNO-B1 red-filter images (Monet et al. 2003). One SN (PTF09fsr) host magnitude was retrieved from the NASA/IPAC Extragalactic Database (NED).$^{13}$ Spectroscopic redshifts were used to calculate absolute magnitudes assuming a cosmological model with $H_{0}=70 \mathrm{~km} \mathrm{~s}^{-1} \mathrm{Mpc}^{-1}$, $\Omega_{m}=0.3$, and $\Omega_{\Lambda}=0.7$. Galactic extinction was removed using the Schlegel et al. (1998) maps via NED. K-corrections are negligible for the typical low redshifts of the sample and were ignored. We adopt a criterion of $M_{r}=-18 \mathrm{mag}$ to distinguish between giant galaxies $\left(M_{r}<-18 \mathrm{mag}\right)$ and dwarf galaxies $\left(M_{r} \geqslant-18 \mathrm{mag}\right)$, based on the observed luminosity distribution (Figure 3 ). This value coincides with physically motivated metallicity thresholds for long-duration GRB occurrence presented by Woosley \& Heger (2006).

The host of PTF09be was not detected in pre-explosion images, but the upper limit on its luminosity indicates that it is a very faint dwarf galaxy. Several host galaxies ${ }^{14}$ subtend a large angular size and were fragmented by the SDSS photometry pipeline. We derived a lower luminosity limit using the cataloged brightness of their core, which places all nine of them in the giant-galaxy class. Finally, one event (PTF09bce) occurred in

\footnotetext{
13 http://nedwww.ipac.caltech.edu/

14 Those of PTF09t, PTF09cjq, PTF09due, PTF09fbf, PTF09hzg, PTF10bau, PTF10bgl, PTF10czn, and PTF10dvb.
}

an area with two possible host galaxies near the line of sight, overlapping each other; it was therefore omitted from the study.

\subsection{Galaxy Metallicities}

In order to enable a comparison with other studies, we must translate our $r$-band luminosities to metallicity values, preferably in solar metallicity units. The procedure for doing this is not straightforward, as it depends on the adopted metallicity scale and the adopted luminosity versus metallicity relation. We obtained metallicity estimates in the following manner. Using the SDSS $g$ - and $r$-band magnitudes of 39 host galaxies for which these data are available, together with the transformation given by Smith et al. (2002), we find the host $B$-band magnitudes. We then use the luminosity versus metallicity relation presented by Tremonti et al. (2004) to find the host metallicities in terms of $\log (\mathrm{O} / \mathrm{H})$. Adopting a solar metallicity of $12+\log (\mathrm{O} / \mathrm{H})=8.9$ (Delahaye \& Pinsonneault 2006), we can then translate our deduced metallicities to the solar scale. At this point, we have metallicity values for 39 host galaxies to which we fit the relation

$$
\log \left(Z / Z_{\odot}\right)=-0.161( \pm 0.012) M_{r}-3.36( \pm 0.23)
$$

(with root-mean square residuals of 0.048 ; the uncertainties of the fit parameters designate a 95\% confidence interval) and use it to estimate the metallicity of all the host galaxies for which $r$-band magnitudes are available.

\section{RESULTS}

A histogram of host $r$-band absolute magnitudes is presented in Figure 3. A bright population centered around $M_{r}=-20 \mathrm{mag}$ can be identified, as well as a faint tail extending to $M_{r}=$ -14 mag. Our choice of $M_{r}=-18$ mag as a limiting value between giant and dwarf galaxies is marked. The number of 

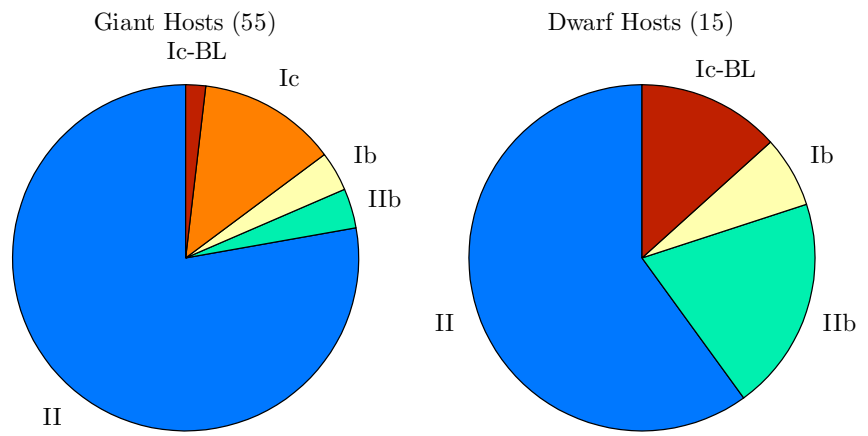

Figure 4. Division of SN types in 55 giant galaxies (left) and 15 dwarf galaxies (right). Note the overabundance of SNe IIb and SNe Ic-BL in dwarf hosts and the lack of "normal" SNe Ic there.

(A color version of this figure is available in the online journal.)

$\mathrm{SNe}$ of each type occurring in giant and dwarf galaxies is listed in Table 2 and shown in Figure 4. Shifting the dwarf/giant cut to $M_{r}=-18.5$ mag increases the dwarf-galaxy sample by three: one SN II, one SN IIb (leaving only one SN IIb in giant hosts), and one SN Ic (making it the only one in a dwarf host under this definition).

\subsection{Caveats}

There are a number of caveats to keep in mind when considering our results.

1. Our analysis is currently limited by the small number of events. This precludes some comparisons with other studies and theoretical predictions, but does allow for significant trends to be identified, as stated below.

2. The difficulty of identifying $\mathrm{SNe}$ in the central core of giant galaxies may induce a bias against $\mathrm{SNe} \mathrm{Ib} / \mathrm{c}$, which are more concentrated in those regions (Anderson \& James 2009). However, Botticella et al. (2008) find that $\sim 50 \%$ of galactic core transients are not SNe. Furthermore, in PTF the difficulty arises only in the central $\sim 1^{\prime \prime}$ of the galaxies (which have much larger typical sizes), so we expect this effect to be negligible (indeed, most nuclear PTF events are active galactic nuclei).

3. In some cases, $\mathrm{SNe}$ can be confused with other transients. For this reason, the spectra of all $\mathrm{SN}$ candidates were checked by eye as well as by using Superfit (Howell et al. 2005) and SNID (Blondin \& Tonry 2007). We retained those candidates which are most likely SNe.

4. Some SNe may have undergone evolution from one subclass to another (for example, from Type II to Type IIb), which could have escaped our notice due to limited spectroscopic coverage. A detailed discussion of this issue will be given in a subsequent paper (A. Gal-Yam et al. 2010, in preparation); however, this uncertainty exists for $\mathrm{SNe}$ regardless of host luminosity and is thus not expected to bias our results systematically.

5. Our derived metallicity values are influenced by the chosen method and adopted parameters. In addition, some nearby GRB hosts have been found to lie below the luminosity versus metallicity relation (i.e., have lower metallicities than their luminosities suggest; Kewley et al. 2007); moreover, while many SNe Ic-BL hosts do lie along the luminosity versus metallicity relation, some outliers have been observed (Modjaz et al. 2008). Using direct host-galaxy measurements could thus be more insightful. However, because
Table 2

Division of SN Types for Each Host-galaxy Class ${ }^{a}$

\begin{tabular}{lccc}
\hline \hline SN Type & No. in Giants (Fraction) & No. in Dwarfs (Fraction) & Likelihood \\
\hline II & $42\left(0.78_{-0.07}^{+0.06}\right)$ & $9\left(0.60_{-0.16}^{+0.14}\right)$ & $6 \%$ \\
IIb & $2\left(0.04_{-0.02}^{+0.05}\right)$ & $3\left(0.20_{-0.11}^{+0.15}\right)$ & $2 \%$ \\
Ib & $2\left(0.04_{-0.02}^{+0.05}\right)$ & $1\left(0.07_{-0.06}^{+0.13}\right)$ & $29 \%$ \\
Ic & $7\left(0.13_{-0.05}^{+0.06}\right)$ & $0\left(0.00_{-0.00}^{+0.05}\right)$ & $7 \%$ \\
Ic-BL & $1\left(0.02_{-0.02}^{+0.04}\right)$ & $2\left(0.13_{-0.08}^{+0.15}\right)$ & $4 \%$ \\
\hline Galaxies & 55 & 15 & \\
\hline
\end{tabular}

Note. ${ }^{\text {a }}$ Two giant-host SNe Ic were counted as half each, due to the possibility of each being a SN Ia. Two out of our sample of $72 \mathrm{SNe}$ were omitted (one due to a classification problem and one because of ambiguous host determination). Fractions are shown with $1 \sigma$ uncertainties, calculated using the Clopper-Pearson method (see Gehrels 1986). The likelihood of both fractions of each SN type arising from the same distribution is displayed, calculated by the method described in Section 4 for the likelihood of the SN IIb and SN Ic-BL overabundances arising by chance.

we do not state any metallicity-specific conclusions in this work, we leave a more detailed treatment of this issue to a future paper.

6. Metallicity varies within galaxies, while here we deal with global metallicity and luminosity values. Indeed, a location study of our SN sample would complement these results.

\section{DISCUSSION}

While small, the number of core-collapse $\mathrm{SNe}$ discovered in dwarf galaxies is higher than expected. Using rough scaling relations to compare PTF with the Pan-STARRS1 predictions presented by Young et al. (2008), it would appear that the number of such $\mathrm{SNe}$ is larger than predicted by a factor of a few. Two of these events, PTF10bfz and PTF10bzf (SN 2010ah; Ofek et al. 2010), are SNe Ic-BL having no obvious association with a GRB. Along with the recent discovery of SN 2007bg (Moretti et al. 2007; Young et al. 2010) as a dwarf-host (Prieto et al. 2008) SN Ic-BL without an associated GRB, ${ }^{15}$ these events suggest that not all SNe Ic-BL in low-metallicity galaxies are associated with long-duration GRBs (cf. Young et al. 2008; Modjaz et al. 2008). However, without direct metallicity measurements, we cannot determine whether they violate the dividing line presented by Modjaz et al. (2008), as these hosts may be outliers to the metallicity versus luminosity relation.

We find that the $\mathrm{SN} \mathrm{Ib} / \mathrm{c}$ to $\mathrm{SN}$ II number ratio is $N(\mathrm{Ib} / \mathrm{c}) / N(\mathrm{II})=0.23_{-0.08}^{+0.11}$ in giant galaxies and $N(\mathrm{Ib} / \mathrm{c}) / N(\mathrm{II})=0.25_{-0.15}^{+0.3}$ in dwarfs $\left(1 \sigma\right.$ uncertainties $\left.{ }^{16}\right)$. While this does not recover the reduction in the $\mathrm{SN} \mathrm{Ib/c} \mathrm{frac-}$ tion in dwarf or low-luminosity hosts reported by Prantzos \& Boissier (2003) and Prieto et al. (2008), the difference is not significant. Theoretical studies (Fryer et al. 2007) predict a fraction of $N(\mathrm{Ib} / \mathrm{c}) / N(\mathrm{II}) \lesssim 0.1$ in dwarf hosts. Distinguishing between $\mathrm{SN} \mathrm{Ib/c} \mathrm{and} \mathrm{SN} \mathrm{II} \mathrm{ratios} \mathrm{of} 0.25$ and 0.1 at the $95 \%$ confidence level requires a dwarf-host SN sample size of $\geqslant 57$ (assuming binomial statistics), which is expected to be attained by PTF within the next few years. The data to be gathered by PTF (as

\footnotetext{
15 Neither SN 2007bg nor PTF10bzf shows radio emissions indicative of the relativistic outflows present in GRBs (Soderberg 2009; Chomiuk \& Soderberg 2010).

16 These confidence levels have been calculated using the Clopper-Pearson
} method, discussed, for example, by Gehrels (1986). 
well as other surveys) will thus allow strong conclusions to be drawn in the near future.

Our distinction among $\mathrm{SNe} \mathrm{Ib}$, Ic, and Ic-BL subtypes, as well as separating Types II and IIb, demonstrates the power of a detailed spectroscopic classification. We find that the only $\mathrm{SNe} \mathrm{Ib} / \mathrm{c}$ in dwarf galaxies are either Ib or Ic-BL. "Normal" $\mathrm{SNe}$ Ic, abundant in large galaxies, are not present, or are rare, in dwarf hosts. This is in agreement with past trends: all $\mathrm{SNe} \mathrm{Ib} / \mathrm{c}$ in dwarfs studied by Prieto et al. (2008) were either SNe Ib (SN 2005hm) or SNe Ic-BL (SN 2006qk, SN 2007I, and SN 2007bg), while all GRB-associated SNe in dwarf hosts are also Ic-BL events (Woosley \& Bloom 2006). Discoveries from the ROTSE-III surveys (Quimby 2006; Yuan et al. 2007) may include one normal SN Ic hosted by a galaxy meeting our definition of a dwarf (SN 2004gk; Quimby et al. 2004), but the sample otherwise shows trends similar to the PTF results.

We further identify an overabundance of $\mathrm{SNe} \mathrm{IIb}$ and $\mathrm{SNe}$ Ic-BL in dwarf galaxies. Assuming the fraction $p$ of SNe IIb is independent of host luminosity, we can calculate the probability of finding $\leqslant 2 \mathrm{SNe}$ IIb in 55 bright $\left(M_{r}<-18 \mathrm{mag}\right.$ ) hosts and $\geqslant 3 \mathrm{SNe}$ IIb in $15 \mathrm{dwarf}\left(M_{r} \geqslant-18 \mathrm{mag}\right)$ hosts. This probability as a function of $p$ is given by

$$
\sum_{x=0}^{2}\left(\begin{array}{c}
55 \\
x
\end{array}\right) p^{x}(1-p)^{55-x} \sum_{y=3}^{15}\left(\begin{array}{c}
15 \\
y
\end{array}\right) p^{y}(1-p)^{15-y} .
$$

Varying $p$ between 0 and 1 , we find that this probability is $<5 \%$. A similar result is found for the overabundance of SNe Ic-BL in dwarf hosts. It is therefore unlikely that the observed trends are produced by chance from the same underlying population.

Our data suggest that "normal" SNe Ic occurring in giant galaxies are replaced by IIb and Ic-BL events in dwarf galaxies. This seems to confirm both trends suggested by the previous location and population-ratio studies, implying two distinct mechanisms at work. Metallicity might be the main process responsible for the evolution of stars which explode as "normal" SNe Ic. In lower metallicity environments, stars that would otherwise explode as SNe Ic end their lives as Ib or IIb events, while many SN Ib progenitors are also driven toward Type IIb explosions following a decrease in their mass loss. However, an additional process which allows for strong stripping in lowmetallicity environments must be present to explain the $\mathrm{SNe}$ Ic-BL and long GRB events observed in dwarf hosts. Binarity and rapid stellar rotation are possible candidates for such a stripping mechanism. Finally, we note an apparent excess of stripped (IIb+Ib+Ic+Ic-BL) SNe in dwarf galaxies, compared to "normal" SNe II (see Figures 3 and 4). If this is indeed the case, it is unlikely to be caused by mass-loss mechanisms, which are expected to be stronger in high-metallicity environments. A top-heavy initial mass function (IMF) in dwarf galaxies might be required to explain this effect (see also Hakobyan et al. 2009; Habergham et al. 2010). Such an IMF is also consistent with pair-production SNe (Gal-Yam et al. 2009) and highly luminous SNe (Quimby et al. 2009), which are associated with very massive progenitors, occurring only in dwarf hosts.

Our analysis is limited by small-number statistics. Continued observations by PTF are expected to rapidly increase the sample size during the coming few years. An attractive complementary study is to analyze the locations of our $\mathrm{SNe}$ within their host galaxies (e.g., as done by Fruchter et al. 2006 and Kelly et al.
2008 on similar-sized samples). A combined analysis should provide a powerful constraint on the evolution and mass-loss properties of massive stars as a function of their metallicity. The distance and angular size of our host sample require the high spatial resolution of the Hubble Space Telescope to carry out this test.

\section{SUMMARY}

We have analyzed the initial compilation of core-collapse $\mathrm{SNe}$ discovered by the PTF during its first post-commissioning year, which comprises the largest homogeneous collection of core-collapse events from a single-untargeted survey ever assembled. Our data indicate an absence of "normal" SNe Ic in dwarf galaxies offset by an excess of SNe IIb and SNe Ic$\mathrm{BL}$. We deduce that a metallicity-dependent process might be responsible for turning "failed SNe Ic" into $\mathrm{SNe} \mathrm{Ib}$ and $\mathrm{IIb}$, while a second process forms the progenitors of SNe Ic-BL and, by association, perhaps also of long-duration GRBs in dwarf galaxies.

Our sample of SNe should increase substantially in the coming years. Ultimately, we plan to analyze the distribution of the different types of $\mathrm{SNe}$ as a function of host-galaxy luminosity, without having to impose an artificial dwarf/giant cut. In addition, high-resolution imaging of host galaxies will enable location studies to be conducted on this SN sample, including (for the first time) a large fraction of dwarf hosts. Such analyses will continue to expand the use of SNe in dwarf galaxies as a powerful tool in the study of massive stars.

The Weizmann Institute PTF partnership is supported by the Israeli Science Foundation via grants to A.G. Collaborative work between A.G. and S.R.K. is supported by the US-Israel Binational Science Foundation. A.G. further acknowledges support from the EU FP7 Marie Curie program via an IRG fellowship, the Benoziyo Center for Astrophysics, a Minerva grant, and the Peter and Patricia Gruber Awards. P.E.N. is supported by the US Department of Energy Scientific Discovery through Advanced Computing program under contract DEFG02-06ER06-04. M.S. acknowledges support from the Royal Society; M.S. and A.G. are also grateful for a Weizmann-UK Making Connections grant. A.V.F. and S.B.C. acknowledge generous support from Gary and Cynthia Bengier, the Richard and Rhoda Goldman Fund, US National Science Foundation grant AST-0908886, and the TABASGO Foundation. J.S.B. was partially supported by a SciDAC grant from the US Department of Energy and a grant from the National Science Foundation (award 0941742). The National Energy Research Scientific Computing Center, which is supported by the Office of Science of the US Department of Energy under contract DE-AC0205CH11231, provided staff, computational resources, and data storage for this project.

The WHT is operated on the island of La Palma by the Isaac Newton Group in the Spanish Observatorio del Roque de los Muchachos of the Instituto de Astrofísica de Canarias. Some of the data presented herein were obtained at the W. M. Keck Observatory, which is operated as a scientific partnership among the California Institute of Technology, the University of California, and NASA; the observatory was made possible by the generous financial support of the W. M. Keck Foundation. We are grateful to the staff of the Keck, Lick, Palomar, Roque de los Muchachos, VLT, and Gemini Observatories for their assistance. We thank Chris Lidman for processing the X-Shooter data used for the classification of PTF10bau. 


\section{REFERENCES}

Abazajian, K. N., et al. 2009, ApJS, 182, 543

Anderson, J. P., \& James, P. A. 2009, MNRAS, 399, 559

Blondin, S., \& Tonry, J. L. 2007, ApJ, 666, 1024

Boissier, S., \& Prantzos, N. 2009, A\&A, 503, 137

Botticella, M. T., et al. 2008, A\&A, 479, 49

Chomiuk, L., \& Soderberg, A. M. 2010, ATel, 2483, 1

Chornock, R., et al. 2010, arXiv:1001.2775

Crockett, R. M., et al. 2008a, MNRAS, 391, L5

Crockett, R. M., et al. 2008b, ApJ, 672, L99

Delahaye, F., \& Pinsonneault, M. H. 2006, ApJ, 649, 529

D’Odorico, S., et al. 2006, Proc. SPIE, 6269, 98

Faber, S. M., et al. 2003, Proc. SPIE, 4841, 1657

Filippenko, A. V. 1997, ARA\&A, 35, 309

Foley, R. J., Smith, N., Ganeshalingam, M., Li, W., Chornock, R., \& Filippenko, A. V. 2007, ApJ, 657, L105

Fruchter, A. S., et al. 2006, Nature, 441, 463

Fryer, C. L., et al. 2007, PASP, 119, 1211

Gal-Yam, A., \& Leonard, D. C. 2009, Nature, 458, 865

Gal-Yam, A., et al. 2005, ApJ, 630, L29

Gal-Yam, A., et al. 2007, ApJ, 656, 372

Gal-Yam, A., et al. 2009, Nature, 462, 624

Gehrels, N. 1986, ApJ, 303, 336

Habergham, S. M., Anderson, J. P., \& James, P. A. 2010, ApJ, 717, 342

Hakobyan, A. A. 2008, Astrophysics, 51, 69

Hakobyan, A. A., Mamon, G. A., Petrosian, A. R., Kunth, D., \& Turatto, M. 2009, A\&A, 508, 1259

Hamuy, M., et al. 2003, Nature, 424, 651

Heger, A., Fryer, C. L., Woosley, S. E., Langer, N., \& Hartmann, D. H. 2003, ApJ, 591,288

Hook, I. M., Jørgensen, I., Allington-Smith, J. R., Davies, R. L., Metcalfe, N., Murowinski, R. G., \& Crampton, D. 2004, PASP, 116, 425

Howell, D. A., et al. 2005, ApJ, 634, 1190

Keller, S. C., \& Wood, P. R. 2006, ApJ, 642, 834

Kelly, P. L., Kirshner, R. P., \& Pahre, M. 2008, ApJ, 687, 1201
Kewley, L. J., Brown, W. R., Geller, M. J., Kenyon, S. J., \& Kurtz, M. J. 2007, AJ, 133,882

Law, N. M., et al. 2009, PASP, 121, 1395

Leloudas, G., Sollerman, J., Levan, A. J., Fynbo, J. P. U., Malesani, D., \& Maund, J. R. 2010, arXiv:1002.3164

Li, W., et al. 2003, PASP, 115, 453

Maund, J. R., Smartt, S. J., \& Schweizer, F. 2005, ApJ, 630, L33

Miller, J. S., \& Stone, R. P. S. 1993, Lick Obs. Tech. Rep., 66

Modjaz, M., et al. 2008, AJ, 135, 1136

Monet, D. G., et al. 2003, AJ, 125, 984

Moretti, S., et al. 2007, IAU Circ., 8834, 1

Ofek, E. O., et al. 2010, CBET, 2198, 1

Oke, J. B., \& Gunn, J. E. 1982, PASP, 94, 586

Oke, J. B., et al. 1995, PASP, 107, 375

Pastorello, A., et al. 2008a, MNRAS, 389, 113

Pastorello, A., et al. 2008b, MNRAS, 389, 955

Prantzos, N., \& Boissier, S. 2003, A\&A, 406, 259

Prieto, J. L., Stanek, K. Z., \& Beacom, J. F. 2008, ApJ, 673, 999

Quimby, R. 2006, PhD thesis, Univ. of Texas

Quimby, R., et al. 2004, IAU Circ., 8446, 1

Quimby, R. M., et al. 2009, arXiv:0910.0059

Rau, A., et al. 2009, PASP, 121, 1334

Schlegel, D. J., Finkbeiner, D. P., \& Davis, M. 1998, ApJ, 500, 525

Smartt, S. J. 2009, ARA\&A, 47, 63

Smith, J. A., et al. 2002, AJ, 123, 2121

Soderberg, A. M. 2009, ATel, 2066, 1

Stanek, K. Z., et al. 2006, Acta Astron., 56, 333

Tremonti, C. A., et al. 2004, ApJ, 613, 898

Tsvetkov, D. Y., Pavlyuk, N. N., \& Bartunov, O. S. 2004, Astron. Lett., 30, 729

van den Bergh, S. 1997, AJ, 113, 197

Woosley, S. E., \& Bloom, J. S. 2006, ARA\&A, 44, 507

Woosley, S. E., \& Heger, A. 2006, ApJ, 637, 914

Young, D. R., Smartt, S. J., Mattila, S., Tanvir, N. R., Bersier, D., Chambers,

K. C., Kaiser, N., \& Tonry, J. L. 2008, A\&A, 489, 359

Young, D. R., et al. 2010, A\&A, 512, 70

Yuan, F., et al. 2007, BAAS, 38, 929 